\section{Partial information and choice behavior in differential reward magnitude learning*}

\author{
DOUGLAS L. MEDIN \\ The Rockefeller University, New York, N.Y. 10021
}

Ss were first trained on a word-number paired-associate list and then given testing on a two-choice differential reward learning paradigm. One of the two words in each pair had been used in the paired-associate training, and the number of points associated with the word was the same as during paired-associate learning. The other member of each pair was new and could have either more or fewer points than the known member of the pair. Speed of learning to ehoose the higher valued member in these pairs was directly related to amount of reward.

The question of what role rewards play in human learning has received active attention since Thorndike first formulated the classic law of effect. A major interpretive problem has revolved around disentangling the informational function as opposed to the satisfying function of rewards.

One situation used in evaluating these two functions of reward is called differential reward magnitude learning. In this paradigm, the $S$ receives concurrent training on a set of problems, each problem consisting of two stimuli (usually common nouns), with a different magnitude of reward being assigned to each alternative. On each trial, the $S$ is permitted to choose one of two stimuli and receives the reward value assigned to that stimulus. In the situations to be considered here, Ss are not informed about the outcome associated with the stimulus not chosen.

In the paradigm described above, one finds a direct relationship between reward magnitude and rate of learning (e.g., Keller, Cole, Burke, \& Estes, 1965) which may appear to provide evidence that rewards have a direct and automatic strengthening function. However, approaches stressing the informational function of reward attribute the direct relationship between choices and reward values to performance effects rather than differences in the rate of forming associations (Humphreys, Allen, \& Estes, 1968), and, indeed, recent research has tended to favor this latter view of reward magnitude learning (see Estes, 1971, for a review).

The basic informational model proposed by Estes and his coworkers (Keller et al, 1965; Humphreys et al 1968) embodies the following assumptions: (1) learning in this situation is a matter of acquiring stimulus-reward associations; (2) these associations are formed in an

*This research was supported by Grants GM 16735 and MH16100 from the U.S. Public Health Service. all-or-none manner, with the probability of an association's occurring being independent of reward magnitude; (3) choices at any point in training are based on the current state of information. The present study sought to obtain more direct evidence concerning the assumption that the rate of forming associations between stimulus alternatives and rewards is independent of reward magnitude. The intent was to bypass the aspects of the model associated with performance differences by giving $S$ s special pretraining. How this was accomplished will become more clear as we consider the details of the model.

The general model assumes that learning proceeds from an initial state, in which the $S$ knows neither stimulus-outcome association, to one of two intermediate states, in which the stimulus-outcome association for either the lower or higher magnitude, but not both, has been learned, and finally to an absorbing state in which stimulus-outcome associations for both choices have been formed. In the initial state, the $\mathbf{S}$ randomly selects an alternative; in the intermediate states, the $S$ chooses the unknown alternative in inverse proportion to the value of known alternative; and in the final state, the $S$ chooses the reward alternative with the higher value.

Because these various learning states are not readily observable in typical reward magnitude experiments, some simplification of this paradigm was attempted. By circumventing the initial stage of learning, the present study was able to obtain more direct evidence concerning the relationship between number of errors (choice of the lower paying alternative) and reward magnitude.
In particular, Ss were given word-number paired-associate training prior to the administration of the differential reward learning situation. The training was designed so that at the beginning of the reward magnitude test, Ss would know the value associated with either the higher or the lower valued stimulus of a pair, i.e., the Ss would be in one of the previously mentioned intermediate learning states. According to the original Keller et al model, this situation can be summarized in terms of the transition matrix in Table 1 , where $\mathrm{C}$ is the probability of forming a stimulus-outcome association and $\alpha$ and $\beta$ are the respective probabilities of switching away from the known stimulus-outcome alternative and choosing the stimulus whose value is unknown from the learned higher state and the learned lower state.

From the matrix, we can clearly see how rewards might affect performance without altering learning. The probability of switching to the stimulus whose value is unknown is inversely related to the value of the known stimulus. In the case where Ss have learned the lower valued alternative, the larger its value, the more reluctant Ss would be to choose the unknown alternative (the smaller $\beta$ would be), and consequently Ss would make more errors. According to the model, the expected total errors would be $(1-\beta) / C$. Therefore, the model predicts that, with the value of the known lower alternative held constant, learning will be independent of the value of the higher, unknown stimulus. When Ss know the higher paying alternative, the expected total errors will be $1 / C$, and therefore independent of both $\alpha$ and $\beta$.

Since we want to consider the possibility that $\alpha$ will be so small for very large known payoffs that a $\mathbf{S}$ would never choose the unknown alternative, our greatest interest will focus on the cases where the value of the higher, known alternative is held constant and we vary the value of the unknown stimulus. According to the model, errors during learning will be independent of the value of the unknown stimulus. Further, given that a $S$ knows the value of the higher stimulus, the model predicts that the expected mean trial of last error will be independent of $\beta$ and equal to $1 / \alpha \mathrm{C}$. Since $\alpha$ is inversely related to the known reward value, this model makes the surprising prediction that

Table 1

\begin{tabular}{|c|c|c|c|c|c|}
\hline & & & ial $n+1$ & & $\begin{array}{c}\text { Probability } \\
\text { Correct }\end{array}$ \\
\hline Trial $\mathbf{n}$ & $\begin{array}{l}\text { Both Learned } \\
\text { Higher Learned } \\
\text { Lower Learned }\end{array}$ & $\begin{array}{l}1 \\
\alpha C \\
\beta C\end{array}$ & $\begin{array}{l}0 \\
1-\alpha \mathrm{C} \\
0\end{array}$ & $\begin{array}{l}0 \\
0 \\
1-\beta C\end{array}$ & $\frac{1}{1}-\alpha$ \\
\hline
\end{tabular}


mean trial of last error should increase directly with the value of the higher valued alternative. Medin (1969) has proposed alternative versions of the general model which do not make this counter-intuitive prediction. However, these variations will not be introduced, since the conclusions from this study will hold for all current versions of the basic reward magnitude learning model.

In brief, in either partial information state, the prediction is made that mean total errors will be independent of the value of the unknown member of a pair. The following experiment provides data bearing on this particular point.

\section{SUBJECTS}

Forty-six college-aged Ss, who reponded to advertisements in a local newspaper, served in the experiment. APPARATUS

Ss were seated in front of an ASR-33 Teletype which was connected to a PDP-8/I computer. Stimulus materials were printed out on the Teletype, and $S s$ responded by pressing keys on the Teletype keyboard. For paired-associate training, single-digit numbers were used for responses; for the differential reward training, Ss selected the word on the left or right by pressing either a key covered with an arrow pointing to the left or a key with an arrow pointing to the right. The computer generated the stimulus materials, controlled the various stimulus and reward sequences, and recorded Ss' responses.

\section{STIMULUS AND \\ REWARD COMBINATIONS \\ Paired-Associate Stimuli}

For paired-associate training, the stimulus members were 12 common four-letter nouns and the response members were the digits 2,4 , or 6 . For a given $\mathrm{S}$, each of the digits was assigned randomly to four of the stimuli.

\section{Differential Reward Training}

The 12 stimuli from paired-associate training, along with their associated response member, were retained for differential reward training. Each of these 12 stimuli was combined with a new common four-letter noun to generate the choice pairs. The values associated with the new stimuli were $1,3,5$, or 7 points, so that the 12 pairs consisted of the following point combinations: $2-1,2-3,2-5,2-7,4-1$, $4-3,4-5,4-7,6-1,6-3,6-5,6-7$. Note that half of the stimuli from paired-associate training were the higher valued members of their pairs while half were the lower valued members for differential reward training. The assignment of point values to the words was determined randomly for each $\mathrm{S}$.

\section{PROCEDURE}

Paired-Associate Learning

Ss were given 16 runs through the 12 item noun-number list. Ss were told to associate the words and numbers and that the correct number would be 2,4 , or 6 . Stimulus orders for each run through the list were randomly determined. A given trial proceeded as follows: (1) the Teletype printed out a word, (2) the $S$ made his response (2, 4 , or 6$),(3)$ The Teletype then printed out the word, along with the number paired with it, and (4) a $1.5 \mathrm{sec}$ intertrial interval ensued. To advance to differential reward training, Ss must have performed perfectly on their last two runs through the list.

Differential Reward Training

Ss were now told that they should consider each number as referring to a number of points and that in this part of the experiment they should try to accumulate as many points as they could. They were further instructed that each word from the first stage of training would have the same number of points associated with it as it had before. Each $S$ received 20 runs through the list of 12 pairs, with the order of each run being randomly determined. On each trial, the words of a pair were printed out, either in a left-right or in a right-left order, the S made his choice, and then the machine printed out the S's choice along with the number of points associated with it. The intertrial interval was $1.5 \mathrm{sec}$. Ss were told they would be paid according to the number of points they accumulated and therefore that they should choose on each trial the word which they thought had more points associated with it. Ss earned between $\$ 2.20$ and $\$ 3.20$, depending on the number of points they totaled, with the average being about $\$ 2.90$. RESULTS

The following results apply for the 40 Ss who met the paired-associate learning criterion. The data from the six noncriterion Ss were not used. In presenting the results, we denote choice of the stimulus of a pair carrying the higher reward value as a correct response and the choice of the stimulus with the lower reward value as an error.

The Trial 1 data bear on the performance assumption of the reward information model that the probability of switching away from a known outcome to a stimulus whose value is not known should be inversely related to the known value. The probability of switching away from a known value of 2,4 , or 6 on Trial 1 was, respectively $.825, .575$, and .244 . We shall presently consider the role these switching probabilities play in modifying performance.

Not all Ss learned to choose the higher paying alternative in each of the pairs. This might happen if a $S$ had a low probability of choosing an unknown stimulus altemative, and, in fact, one $\mathbf{S}$ never deviated from the paired-associate training stimuli, making either all correct responses or all errors for a given pair. More importantly, there was a trend for the probability of Ss settling on uniform choice of the initially known lower alternative to be inversely related to the value of the initially unknown higher alternative. Thus, in the $2 \cdot 3$, $2-5$, and $2-7$ pairs, 6,2 , and 2 protocols (out of 40) exhibited choices of the lower alternative on each of the last 10 trials, and in the 4-5 and 4-7 pairs, the corresponding numbers of protocols absorbed on the lower alternative were 8 and 3 .

Mean total errors and mean trial of last error shown in Table 2 reveal more directly a fundamental shortcoming of the assumption that rate of learning is independent of the value of the unknown stimulus. The table eliminates those protocols where Ss never chose the unknown member of a pair, but including those protocols would in no way alter our conclusions. Given a particular value of an initially known lower alternative, mean errors and mean trial of last error decreased as the value of the higher member of a pair increased. Individual t tests on errors showed that only the 2-5, 2-7 comparison failed to achieve statistical reliability.

With the value of an initially known higher alternative held constant, mean trial of last error and mean errors generally were directly related to the value of the unknown lower alternative. Additional individual $t$ tests on mean total errors showed that only the 6-3, 6-5 comparison failed to reach statistical reliability. These data directly contradict the assumption of the model that mean errors will be independent of the value of the unknown member of a stimulus pair.

It appears that the relationships in the upper diagonal of Table 2 are stronger than the relationships in the lower diagonal. However, statistically speaking, the relationships in the lower diagonal tend to be more reliable. This is partially related to the definition of an error. If a few Ss were very reluctant to choose the unknown stimulus alternative, this tendency would greatly increase the variability of the total errors in the upper diagonal. Much of the difference between performance on $2-7,4-7$, and 6-7 pairs can be attributed to differing probabilities of switching away from choosing a known value of 2,4 , or 6 .

This same interpretation can account for the fact that mean trial of 
the last error was greater for the $6 \cdot 1$, $6-3$, and $6-5$ pairs than for the $2-1,4-1$, and 4-3 pairs. According to the value derived from the reward information model for the mean trial of last error, $1 / \alpha \mathbf{C}$, mean trial of last error should be directly related to the value of the known higher value. This would occur because Ss might be reluctant to switch from a known alternative of high value, with the result that errors might not occur until relatively late in testing (if at all). This outcome occurred in the face of other factors (e.g., absorptions) which might have militated against this relationship.

$$
\text { DISCUSSION }
$$

The results of this study run strongly against the assumption that rate of learning is independent of reward magnitude. With knowledge concerning one stimulus value controlled, total errors and trial of last error were systematically related to the unknown stimulus values. These relationships appear immediately and are prominent. Consider, for example, the pairs involving a known stimulus value of 4 . Given that a $S$ chose the unknown alternative on Trial 1 (which occurred $57.5 \%$ of the time), he then avoided the known value of 4 on Trial $220.8 \%, 47.8 \%, 81.0 \%$, and $91.7 \%$ of the time for the $4-1,4-3,4-5$, and 4-7 pairs, respectively. It seems, then, that some revision of the original model is in order.

In a separate attempt to obtain direct observations of the states of learning assumed by the reward magnitude model, Allen \& Estes (1972) required Ss to estimate the value of the stimulus alternative they chose during reward magnitude learning. Allen and Estes found that, contrary to the assumptions of the general model, the rate of acquisition of correct value estimates was systematically related to reward value and, further, that there was a significant improvement in choosing the higher valued alternative of a stimulus pair before the first evidence appeared that a $\mathbf{S}$ had learned either of the reward values. They suggested the possibility that Ss employed differential rehearsal of stimulus-outcome associations, rehearsing higher valued alternatives more than lower valued associations. This would tend to increase the probability that a stimulus-outcome association would be recalled, and failing that, the rehearsed stimulus would tend to be more familiar. Given that Ss choose the more familiar stimulus in the absence of a specific stimulus-outcome association, performance would be modified by reward before $\mathrm{Ss}$ had a stimulus-outcome association available in long-term memory (as Allen and

Table 2

Adjusted Mean Errors and Trial of Last Error (Eliminating Protocols Where Ss Failed Ever to Choose the Unknown Altermative)*

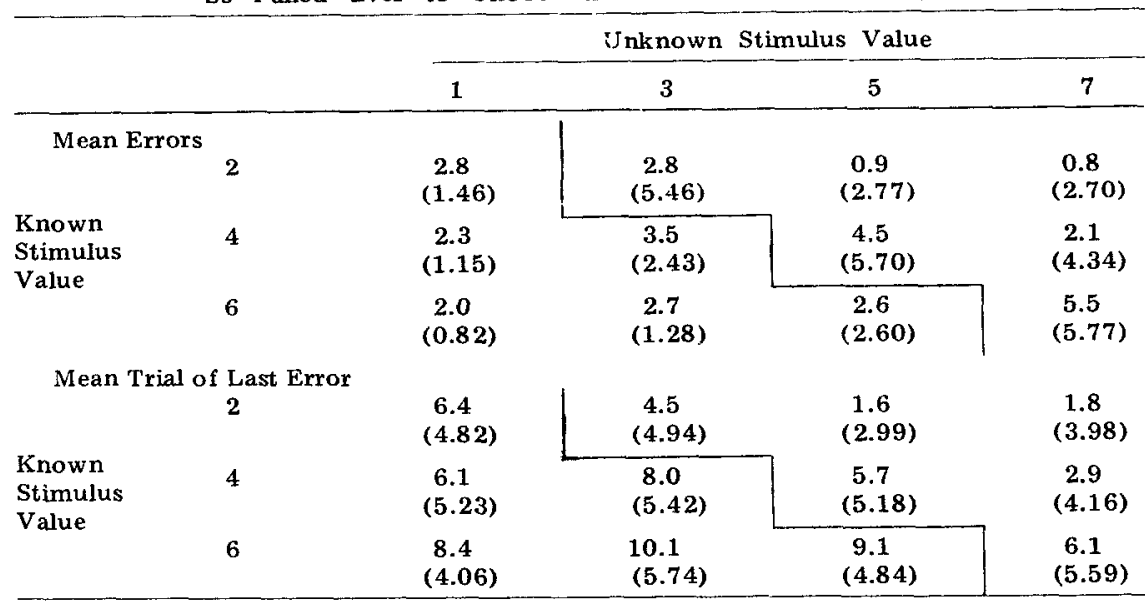

*The values in the upper diagonal involve unknown values higher than the known.value while in the lower diagonal the known value is higher than the unknown value. The values in parentheses are standard deviations.

Estes found). From a S's point of view, he should choose the most familiar stimulus because he probably rehearsed that stimulus-outcome association; and if he did rehearse it, this was probably because the stimulus was associated with a relatively good outcome.

Three lines of evidence suggest that some form of differential rehearsal and use of familiarity or frequency may have occurred in the present experiment. First, the similarity of this study to that of Allen and Estes encourages a similar interpretation. In addition, results from verbal discrimination learning experiments which might be considered as a special case (rewards of 1 vs 0 ) of differential reward learning have provided considerable support for a model which assumes that differential frequency is the primary basis of choice behavior (Eckstrand, Wallace, \& Underwood, 1966). Finally, Ss in the present experiment said that they rehearsed stimuli by themselves rather than stimulus-outcome associations. This latter observation suggests that frequency may play a direct rather than an auxiliary role in Ss' choice behavior. That is, Ss reported rapidly rehearsing the stimulus itself, with the amount of rehearsal roughly related to the value of the outcomes. If familiarity serves as a reliable cue, this strategy would be efficient, since, for example, a S could rehearse "fire" more rapidly and thus more often than "fire-seven."

How might frequency be incorporated into a model to fit the present data? First of all, it is clear that frequency alone is inadequate. Since all the stimuli from the paired-associate training would be approximately equal in familiarity, frequency by itself could not account for the finding that the probability of switching away from the known stimulus value on Trial 1 was inversely related to its value. Reward information must have some function in an adequate model for the current data.

A second alternative would be to assume that $\mathrm{Ss}$ use the information from paired-associate training to control their differential rehearsal and use of frequency. One possibility is that if the $\mathrm{S}$ chooses the unknown alternative which has a value higher than the known stimulus, he rehearses the unknown alternative a number of times directly related to its value. If he chooses the unknown alternative and it has a value lower than that of the known stimulus, he rehearses the known stimulus a number of times directly related to the value of the known stimulus. This scheme would fail since it will not predict the finding that the 4-1 combination was learned faster than 4-3, and the 6-1 pair was learned faster than 6-3 and 6-5. If a $S$ rehearsed the unknown lower value, one might expect an increased probability of an error on the next trial, which clearly did not occur. For example, the $57.5 \%$ probability of choosing the unknown alternative when the known value is 4 on Trial 1 changes to $20.8 \%$ after Ss received an outcome of value 1 in the 4-1 pairs.

A more viable possibility attributes still more use of reward information in producing frequency differences. According to this idea, after a choice is made, the $S$ rehearses the higher stimulus alternative (which he can infer from the known outcome) in direct proportion to his expected gain 
for doing so. Thus, given a higher known value of 4 , the $S$ might rehearse the higher value more when he chooses the lower alternative and receives a 1 as his outcome than when he receives a 3 as his outcome. A S might rehearse a stimulus whose value is 5 in a $2-5$ pair considerably when he chooses it but not rehearse the 5 at all in a 6-5 pair. Therefore, one would expect errors to be related to the value of the unknown alternative regardless of whether it was the higher or lower alternative, and this relationship would produce an inverse function between errors and reward differences within a stimulus pair. In the absence of a distinct frequency or familiarity difference, $S s$ might be expected to choose the unknown stimulus in some direct proportion to their expected probability of gaining by switching, which would tend to be large for a small known value and small for a large known value. It is still assumed that Ss can form new stimulus-outcome associations, much as they did in paired-associate training. However, since we assume that Ss rehearse stimuli rather than full stimulus-outcome associations, these new stimulus-outcome associations would play only an auxiliary role in the test situation, being most likely to be formed after $S$ s were reliably choosing a higher unknown stimulus.

There still remains one major problem. What about all the frequency or familiarity accrued during the preliminary paired-associate training? It would seem that this frequency would tend to overwhelm any effects of frequency produced by differential rehearsal on a given trial. This must certainly be true if frequency information is based on some unitary trace, varying only in strength. Fortunately for the current model, Hintzman \& Block (1971) have recently provided evidence that $\mathrm{Ss}$ can discriminate between recent and remote frequencies of a word. In one experiment, words appeared 0,2 , or 5 times in one list, a 5-min rest interval intervened, and then the same words appeared 0,2 , or 5 times in a second list. Ss were asked to estimate the number of times a word had occurred in a given list. Although there was some influence of word frequency in one list on estimated frequency in the other list, Ss were quite efficient in judging frequency within a particular list. As applied to the present study, this would suggest that Ss could largely ignore frequencies built up in paired-associate pretraining when responding on the differential reward magnitude trials, which began approximately $3 \mathrm{~min}$ after the paired-associate training. This result is certainly plausible, since for frequency to exert any influence on choices between these very familiar words, Ss must ignore substantial extraexperimental frequency differences accumulated over an individual's lifetime.

Although the results of the present study are consistent with the idea that Ss use reward information to control differential rehearsal, more direct evidence in favor of these ideas is needed. Assuming for the moment that Ss employ both rehearsal and choice of familiar stimuli, the immediate task for theories of choice learning becomes to specify the role of familiarity and stimulus-outcome associations in differential reward learning more precisely in order to obtain some notion of their relative importance. In the case of frequency, one would like to know how context-specific frequency information may be (i.e., which contextual cues are effective) and whether frequency accumulated by rehearsal is distinct from frequency accumulated by seeing a word printed out on the Teletype. A second consideration concerns what experimental structures might tend to give rise to rehearsing just stimuli as opposed to stinulus-outcome associations.

In the version of the model giving the best account of the current data, frequency emerges as an important cue. But even here it is clear that the variables assumed to give rise to frequency differences are neither direct nor autonomatic in their effect. It seems likely that information will continue to play a major role in interpretations of the function of reward in human learning.

\section{REFERENCES}

ALLEN, G. A., \& ESTES, W. K. Acquisition of correct choices and value judgments in binary choice learning with differential rewards. Psychonomic Science, 1972, in press.

ECKSTRAND, B. R., WALLACE, W. P., \& UNDER WOOD, B. J. A frequency theory of verbal discrimination learning. Psychological Review, 1966, 73, 566-578.

ESTES, W. K. Reward in human learning: Theoretical issues and strategic choice points. In R. Glazer (Ed.), The nature of reinforcement. New York: Academic Press, 1971. Pp. 16-36.

HINTZMAN, D. L., \& BLOCK, R. A. Repetition and memory: Evidence for a multiple trace hypothesis. Journal of Experimental Psychology, 1971, 88. 297-306.

HUMPHREYS, M. S : ALLEN, G. A., \& ESTES, W. $K$. Learning of two-choice, differential reward problems with information constraints on payoff combinations. Journal of Mathematical Psychology, 1968, 5, 260-280.

KELLER, L., COLE, M., BURKE, C, J., \& ESTES, W. K. Reward and information values of trial outcomes in paired-associate learning. Psychological Monographs, 1965, 79(12, Whole No. 605 ).

MEDIN, D. L. Models for two-choice differential reward learning. Technical Report, The Rockeriller University, 1969 . 